\title{
Metabolomics in Cardiovascular Diseases: Biomarkers Quest
}

\section{Ali Alawieh, Firas H Kobaissy, Mazen Kurban and George Nemer*}

\section{Department of Biochemistry and Molecular Genetics, American University of Beirut, Beirut, Lebanon}

Cardiovascular Diseases (CVD) remain the leading cause of death and reduced life expectancy worldwide according to the 2010 reports of the Global Burden of Disease [1,2]. CVDs affect all age groups, and the number of affected individuals is becoming more alarming, thus the importance of defining early diagnostic biomarkers, that can be helpful to detect and possibly prevent the occurrence of cardiovascular diseases and associated accidents, is of utmost importance. Ultimately these biomarkers could help establishing a personalized regimen for the treatment of a given patient and open the way for a novel drug design strategy.

Several biomarker discovery approaches have been incorporated into the field of CVD, but of recent interest is the application of metabolomics approach. The metabolome is defined as the total complement of low-molecular-weight metabolites in a biological system under a set of environmental conditions [3,4]. These metabolites can be the direct result of the individual's genome; thus, forming the primary metabolome or can include the byproducts of the metabolism of commensal bacteria living in the body forming the secondary metabolome. Both primary and secondary metabolomes are being investigated in the quest of biomarker discovery for CVD [4]. Metabolomics application on serum samples has been of increasing interest in CVD given that heart tissue is not available for investigation as with malignancy, for instance. The serum study of metabolites can provide a min-to-min profile of the metabolic changes that can help detect the adaptive "metabolic shift" that cardiomycoytes undergo in several CVD. This shift is reflected by changes in terms of metabolite utilization and modification. These changes could be an early sign of the perturbations associated with CVD. Studying metabolic profiles in CVD is of increasing interest given that metabolites are the "proximal receptors" of disease phenotypes [5].

Similar to other biomarker deciphering disciplines, metabolomics aims at discovering prognostic and diagnostic biomarkers of CVD. These would be used for better risk assessment, early detection of the disease and limiting the use of invasive expensive and time-consuming procedures such as angiography, and finally monitoring disease progression and response to treatment [6]. As these biomarkers are predicted to be central hubs in the systems' metabolic network, they would also provide an insight into the pathogenesis of these diseases.

Two core technologies have been used in plasma metabolite profiling: Nuclear Magnetic Resonance spectroscopy (NMR) and tandem Mass Spectrometry (MS). Each technique has its own limitations and advantages. NMR requires small sample amounts and is nondestructive allowing for detection of metabolites in intact tissue and even in vivo [7]. However, NMR suffers from low sensitivity and the ability to detect only high abundance metabolites. MS should be coupled to a method of separation of the analytes as Liquid Chromatography (LC) or Gas Chromatography (GC). MS has a higher sensitivity but leads to the destruction of the sample [8,9]. These highthroughput techniques can be used in horizontal studies involving comparison of patients to controls or in longitudinal studies as with monitoring subtle changes of a sample over time.

Investigators in metabolomic research invariably adopt one of two strategies, a closed profile targeted strategy or an open profile pattern discovery strategy [4]. The former monitors a limited number of metabolites that are previously known and is therefore hypothesisdriven. Targeted profiling is considered a biased approach; however, it allows for easy analysis of the metabolites, have better reproducibility and can be used to validate biomarkers after their discovery. The pattern-discovery approach assays all the detectable metabolites in a sample, and is therefore hypothesis-generating. Investigators use pattern-discovery approach to mine raw data for biological hypotheses and to get an insight into new pathways involved in the disease. This approach is less-biased but is by far less reproducible since different conditions and confounders may not be accounted for.

Applied to CVD research, metabolomics were used to determine serum metabolic profiles associated with coronary artery disease (CAD) [10-12], myocardial ischemia [13-17] heart failure [18], atrial fibrillation [19], atherosclerosis [20] and others. The majority of the groups studying metabolomics of CVD adopted the pattern discovery; however, the group of Shah et al. [10] was one of the prominent groups working on targeted metabolomics in peripheral blood samples of patients with CAD. The group first demonstrated the heritability of metabolic profiles in CAD in a pilot study [10]. The study involved the use of targeted quantitative mass spectrometry for the metabolomic profiling of 66 metabolites among 117 individuals from GENECARD study that involved families with siblingsof premature CAD. High heritabilities were associated with metabolites like amino acids (arginine, ornithine, alanine, proline, leucine/isoleucine, valine, glutamate/glutamine, phenylalanine and glycine), free fatty acids (arachidonic, palmitic, linoleic), and acylcarnitines (especially long-chain). Additionally, Shah et al. [10] demonstrated the association of similar metabolic profiles with CAD and cardiovascular events using the same targeted MS/MS techniques. Target metabolites included acylcarnitines, amino acids, and other metabolites. Subjects were chosen from the CATHGEN biorepository to evaluate suspected ischemic heart disease. CATHGEN includes subjects undergoing cardiac catheterization at Duke University Medical Center. The study was highly significant as it involved a large sample size, controlled for the different factors that could modulate the metabolic profiles (drugs, medical conditions), and used an initial and replicative sample groups. The study demonstrated significant variation between cases and controls for amino like acids leucine/ isoleucine, valine, glutamate/glutamine, proline, and methionine, as well as acylcarnitines. This difference persisted for most metabolites

*Corresponding author: Georges Nemer, Department of Biochemistry and Molecular Genetics, American University of Beirut, Bliss Street, Beirut, Lebanon, E-mail: gn08@aub.edu.lb

Received January 17, 2013; Accepted January 17, 2013; Published January 22 2013

Citation: Alawieh A, Kobaissy FH, Kurban M, Nemer G (2013) Metabolomics in Cardiovascular Diseases: Biomarkers Quest. J Data Mining Genomics Proteomics S2: e001. doi:10.4172/2153-0602.S2-e001

Copyright: ( 2013 Alawieh A, et al. This is an open-access article distributed under the terms of the Creative Commons Attribution License, which permits unrestricted use, distribution, and reproduction in any medium, provided the original author and source are credited. 
after adjustment for CAD risk factors [10]. The study provided a cluster of metabolites that are associated with CAD and can predict clinical events in patients having the disease. Such cluster could help discriminate patients with $\mathrm{CAD}$ and provide better risk stratification of these patients especially that such cluster provides a predictive value beyond the available risk factors. It also supported the implication of several pathways in the pathogenesis of CAD including alterations in mitochondrial fatty acid oxidation, carboxylation at the endoplasmic reticulum, or peroxisomal metabolism [10]. The association between acyl carnitines and CVD has been also studied at the genome level. Our group has described a founder mutation (R254X) in the SLC22A5 gene to be responsible for the Primary Carnitine Deficiency (PCD) causing dilated cardiomyopathy in three families in Lebanon whose members recovered with the administration of L-Carnitine [21].

Other groups used pattern discovery approach to assay for metabolic profiles associated with CVD. Wang et al. [12] used Liquid chromatography with on-line mass spectrometry to generate unbiased small-molecule metabolic profiles in plasma to predict the risk for CVD. The study involved profiling more than 2000 metabolites. Three of these metabolites, namely choline, Trimethylamine $\mathrm{N}$-oxide (TMAO) and betaine, were shown to predict the risk for CVD in a large clinical cohort. These three metabolites are products of the breakdown of dietary phospholipids. This study implicates phospholipids in the pathogenesis of CVD as well as a role for cholesterol, triglycerides and sphingolipids [22]. The group confirmed their results using mouse models where the dietary supplementation of choline, TMAO or betaine promoted up regulation of multiple macrophage scavenger receptors linked to atherosclerosis. The study additionally demonstrated the relationship between the metabolism of phosphatidylcholine by gut-flora metabolism and CVD pathogenesis. Phospholipid profiles were also associated with myocardial ischemia in a study by Lin et al. [14] using ultra-performance liquid chromatography coupled with quadrupole-time-of-flight. Results demonstrated that 4 kinds of phospholipids showed tight relationship with the occurrence of Silent Myocardial Ischemia [14]. Early serum metabolic changes in myocardial ischemia were assessed by two studies involving an in-vivo simulation of myocardial ischemia. The first by Lewis et al. [5], Lewis et al. [15] who used an artificial human model planned myocardial infarction (PMI) in the setting of alcohol septal ablation treatment for patients with hypertrophic obstructive cardiomyopathy. Results provided a PMI-derived metabolic signature consisting of aconitic acid, hypoxanthine, trimethylamine $\mathrm{N}$-oxide, and threonine that differentiated patients with silent myocardial ischemia from those undergoing diagnostic coronary angiography. The second study by Bodi et al. [16] investigated a metabolic bio-signature of myocardial ischemia using NMR on serum of patients under angioplasty balloon induced transient coronary occlusion. The resulting signature applied to group of patients with chest pain had positive predictive value of $75 \%$ and negative predictive value of $96 \%$ [16].

Metabolic profiles were also studied as an alternative to the exercise stress test in patients not capable of performing it. Sabatine et al. [17] quantified 500 metabolites released after exercise-induced myocardial injury using LC-MS. Serum samples before and after exercise stress testing were compared between patients showing myocardial ischemia and those who didn't based on clinical guidelines [17]. The group identified changes in the levels of 6 components of the citric acidcycle that were significantly overrepresented among the metabolites that changed specifically with myocardial ischemia. A similar study was performed by Barba et al. [13] using NMR to define a predictor for exercise-induced myocardial ischemia. The metabolic profile included among others, lactate glucose and long chain amino acids, and was able to predict $84 \%$ of the cases [13].

In studying the metabolomics of heart failure, Dunn et al. [18] used GC-MS technique to profile the metabolites of 52 subjects with systolic heart failure in an attempt to identify new biomarkers for HF, other than the well established BNP (brain natriuretic protein) biomarker. The study detected 38 metabolites of high significance in a pool of 272 candidate metabolites. Of major interest were two metabolites, pseudouridine and 2-oxoglutarate, that showed similar predictive results to BNP. Pseudouridine is a modified nucleoside that is found in ribosomal and transfer RNA and is produced post-transcriptionally. 2-oxoglutarate is a major intermediate of the tri-carboxylic-acid cycle and is associated with energy metabolism. These two metabolites have not previously been associated with $\mathrm{HF}$ and provide a new insight on the pathophysiology of the disease that requires further analysis.

Despite these different attempts to get potential metabolic biomarkers of CVD into clinical application, none has made it to the diagnostic laboratories. The current markers are still of limited reliability until validated by multiple studies to characterize their clinical application [6]. Similar to proteomics and genomics, several false discoveries are associated with metabolomic investigation. Metabolomic tools are still not mature as the field is still in its infancy. The characterization of the human serum metabolome is ongoing and the most recent study, Psychogios et al. [23] reports 4229 serum metabolites. The field of metabolomics also awaits major advances in discovery of techniques as well as software and data analysis tools to overcome the limitations of metabolic investigation Such limitations include the lack of amplification techniques for metabolites, the high chemical diversity among molecules, the differences in half lives and the high dynamic range [7]. Metabolomic pattern discovery strategies should be well adjusted to confounders to further enhance specificity and reproducibility. One step in this attempt was performed by Brunner et al. [24] who used mass spectrometry and conventional immunoassays to determine the effect of heparin on the metabolic profile. The study defined a metabolite signature related to heparin administration to be used in the interpretation of the results of metabolic profiling in patients taking heparin [24]. Finally, as with other omics-discovery fields, metabolomics will probably have a great impact in the coming years by combining it to genomic and proteomic.

\section{References}

1. Lozano R, Naghavi M, Foreman K, Lim S, Shibuya K, et al. (2013) Global and regional mortality from 235 causes of death for 20 age groups in 1990 and 2010: a systematic analysis for the Global Burden of Disease Study 2010 Lancet 380: 2095-2128.

2. Murray CJ, Vos T, Lozano R, Naghavi M, Flaxman AD, et al. (2013) Disabilityadjusted life years (DALYs) for 291 diseases and injuries in 21 regions, 19902010: a systematic analysis for the Global Burden of Disease Study 2010 Lancet 380: 2197-2223.

3. Mayr M (2008) Advances in Genetics, Proteomics, and Metabolomics

4. Senn T, Hazen SL, Tang WH (2012) Translating Metabolomics to Cardiovascular Biomarkers. Prog Cardiovasc Dis 55: 70-76.

5. Lewis GD, Asnani A, Gerszten RE (2008) Application of metabolomics to cardiovascular biomarker and pathway discovery. J Am Coll Cardiol 52: 117123.

6. Dunn WB, Goodacre R, Neyses L, Mamas M (2011) Integration of metabolomics in heart disease and diabetes research: current achievements and future outlook. Bioanalysis 3: 2205-2222.

7. Heather LC, Wang X, West JA, Griffin JL (2012) A practical guide to metabolomic profiling as a discovery tool for human heart disease. J Mol Cell Cardiol.

8. Lewis GD, Gerszten RE (2010) Toward metabolomic signatures of cardiovascular disease. Cir Cardiovasc Genet 3: 119-121. 
Citation: Alawieh A, Kobaissy FH, Kurban M, Nemer G (2013) Metabolomics in Cardiovascular Diseases: Biomarkers Quest. J Data Mining Genomics Proteomics S2: e001. doi:10.4172/2153-0602.S2-e001

9. Gerszten RE, Wang TJ (2008) The search for new cardiovascular biomarkers Nature 451: 949-952.

10. Shah SH, Hauser ER, Bain JR, Muehlbauer MJ, Haynes C, et al. (2009) High heritability of metabolomic profiles in families burdened with premature cardiovascular disease. Mol Syst Biol 5: 258

11. Shah SH, Bain JR, Muehlbauer MJ, Stevens RD, Crosslin DR, et al. (2010) Association of a peripheral blood metabolic profile with coronary artery disease and risk of subsequent cardiovascular events. Circ Cardiovasc Genet 3: $207-$ 214.

12. Wang Z, Klipfell E, Bennett BJ, Koeth R, Levison BS, et al. (2011) Gut flora metabolism of phosphatidylcholine promotes cardiovascular disease. Nature 472: 57-63.

13. Barba I, de León G, Martín E, Cuevas A, Aguade S, et al. (2008). Nuclear magnetic resonance-based metabolomics predicts exercise-induced ischemia in patients with suspected coronary artery disease. Magn Reson Med 60: $27-$ 32.

14. Lin $\mathrm{H}$, Zhang J, Gao $P$ (2009) Silent myocardial ischemia is associated with altered plasma phospholipids. J Clin Lab Anal 23: 45-50.

15. Lewis GD, Wei R, Liu E, Yang E, Shi X, et al. (2008) Metabolite profiling of blood from individuals undergoing planned myocardial infarction reveals early markers of myocardial injury. J Clin Invest 118: 3503-3512.

16. Bodi V, Sanchis J, Morales JM, Marrachelli VG, Nunez J, et al. (2012) Metabolomic profile of human myocardial ischemia by nuclear magnetic resonance spectroscopy of peripheral blood serum: a translational study based on transient coronary occlusion models. J Am Coll Cardiol 59: 1629-1641.
17. Sabatine MS, Liu E, Morrow DA, Heller E, Mccarroll R, et al. (2005) Metabolomic identification of novel biomarkers of myocardial ischemia. Circulation 112 3868-3875.

18. Dunn WB, Broadhurst DI, Deepak SM, Buch MH, McDowell G, et al. (2007) Serum metabolomics reveals many novel metabolic markers of heart failure, including pseudouridine and 2-oxoglutarate. Metabolomics 3: 413-426.

19. De Souza Al, Cardin S, Wait R, Chung YL, Vijayakumar M, et al. (2010) Proteomic and metabolomic analysis of atrial profibrillatory remodelling in congestive heart failure. J Mol Cell Cardiol 49: 851-863.

20. Chen X, Liu L, Palacios G, Gao J, Zhang N, et al. (2010) Plasma metabolomics reveals biomarkers of the atherosclerosis. J Sep Sci 33: 2776-2783.

21. Yamak A, Bitar F, Karam P, Nemer G (2007) Exclusive cardiac dysfunction in familial primary carnitine deficiency cases: a genotype-phenotype correlation. Clin Genet 72: 59-62.

22. El Alwani M, Usta J, Nemer G, El Sabban M, Nasser M, et al. (2005) Regulation of the sphingolipid signaling pathways in the growing and hypoxic rat heart Prostaglandins Other Lipid Mediat 78: 249-263.

23. Psychogios N, Hau DD, Peng J, Guo AC, Mandal R, et al. (2011) The human serum metabolome. PLoS One 6: e16957.

24. Brunner MP, Shah SH, Craig DM, Stevens RD, Muehlbauer MJ, et al. (2011) Effect of heparin administration on metabolomic profiles in samples obtained during cardiac catheterization. Circ Cardiovasc Genet 4: 695-700.
This article was originally published in a special issue, Systems Biology: Biomarker Discovery handled by Editor: Dr. Firas Kobaissy, University of Florida, USA 